\title{
MAIN GENOME EDITING TOOLS: AN OVERVIEW OF THE LITERATURE, FUTURE APPLICATIONS AND ETHICAL QUESTIONS
}

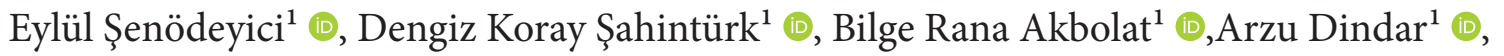

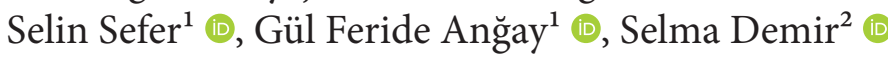

${ }^{1}$ Trakya University School of Medicine, Edirne, TURKEY

${ }^{2}$ Department of Medical Genetics, Trakya University School of Medicine, Edirne, TURKEY

\begin{abstract}
The popularity of genome editing technologies in the scientific community has been on the rise for several years. These technologies are slowly becoming a ray of hope for many patients with genetic diseases, thanks to their immense potential for clinical application. New genome editing tools are being rapidly developed and introduced, while pre-existing ones are being perfected. In the process beginning with the completion of the Human Genome Project to the first clinical trials focusing on cancer immunotherapy and treating blindness, studies on genome editing have increased exponentially. The clustered regularly interspaced short palindromic repeats system is a Nobel Prize-winning genome editing tool celebrated by many researchers and is often praised due to its ease of use, low cost, and efficiency compared to other acknowledged genome editing tools. This review aims to discuss the historical development, working mechanisms, present and future clinical applications of zinc-finger nucleases, transcription activator-like effector nucleases, clustered regularly interspaced short palindromic repeats, and prime editors, while presenting the ethical aspects of using these genome editing tools. Keywords: Zinc-finger nucleases, transcription activator-like effector nucleases, clustered regularly interspaced short palindromic repeats, gene editing
\end{abstract}

\section{INTRODUCTION}

In the last decades, a multitude of novelties was introduced to the field of genetics including the first gene transfer into mammalian cells, cloning of the insulin gene, production of human recombinant lysosomal enzymes, and sequencing the human genome (1-4). Completion of the Human Genome Project provided researchers with a deeper understanding of the role of genetics in physiology and evolution of humankind, the entirety of the human genome, and how genes function, along with the possibility to do systematic research on causes of diseases, which significantly altered the practice of medicine $(5,6)$. Following this enlightenment, several gene therapy techniques aiming to mitigate the disease-causing effects of genetic conditions have been developed, namely zinc-finger nuclease (ZFN), transcription activator-like effector nuclease (TALEN), and clustered regularly interspaced short palindromic repeats (CRISPR) (7-9). These are programmable site-specific nucleases that have shown therapeutic potential; however, the applications of these technologies are still being perfected. Although CRISPR/CRISPR associated protein 9 (Cas9) systems are reported to have shown superiority over transcription activator-like effector nucleases (TALENs) and zinc-finger nucleases (ZFNs) on certain aspects, effects of off-target mutations remain untackled and several ethical questions remain unanswered $(7,10)$.

\section{Zinc-Finger Nucleases}

Induction of a double-strand break (DSB) in a specific genomic target sequence, followed by the generation of desired modifications during subsequent DNA break repair enabled researchers to specifically add or delete genetic information to study gene function in different organisms. ZFNs are artificially engineered restriction enzymes with a hybrid heterodimeric protein structure that can be customized to cleave a targeted DNA region and they are among the first tools of genome editing technology (Figure 1). The FokI endonuclease domain induces double-strand breaks (DSBs) whereas zinc-finger domains are responsible for DNA identification (11). The FokI cleavage domain resides within a 5 or 6 base pair (bp) long spacer sequence, with engineered Cys2His2 zinc-fingers and a linker protein that creates a connection between FokI and zinc-fingers $(12,13)$.

FokI, a separable enzyme, requires to be dimerized for cleavage; however, the dimer interface cannot maintain enough strength. The best way to overcome this problem is to construct a second set of fingers at the opposite position. Each monomer determines a half site with three or four Cys2His2 zinc-fingers that were first detected in the genus Xenopus, and each zinc-finger binds to a 3 bp sequence of DNA $(12,13)$. Dimerization appears as a powerful disadvantage; however, cleavage gets activated when there is enough specificity, and two domains can reconstruct it (14). 
Studies on ZFN presents hope for a cure for infections such as the human immunodeficiency virus (HIV), human papillomavirus, hepatitis B virus (HBV), and malaria (15-19). In 2009, Kim et al. (20) tested 315 modularly assembled ZFN pairs at 33 sites in the CC chemokine receptor 5 (CCR5) gene, which is vital for curing acquired immune deficiency syndrome (AIDS). Knocking out CCR5 leads to providing insistent T-cells for HIV infection and insertions at CCR 5 are considered safe sites for gene therapy. Deleting the CCR5 gene concludes with the repair of the luciferase gene in human embryonic kidney cells (HEK293). Therefore, the activity of the luciferase gene is an indicator of cleavage success.

Context-dependent specificity is noted as one of the most prominent downsides of ZFNs, meaning that the nucleases' capability of cleaving the target sequence is affected by the adjacent sequences in the genome and the target sequence. Instability and genome fragmentation may be caused by the occurrence of these non-specific cleavages. Additionally, ZFN induced non-specific and off-target cleavages may also cause toxicity to cells, making this technique undesirable for several practices (11).

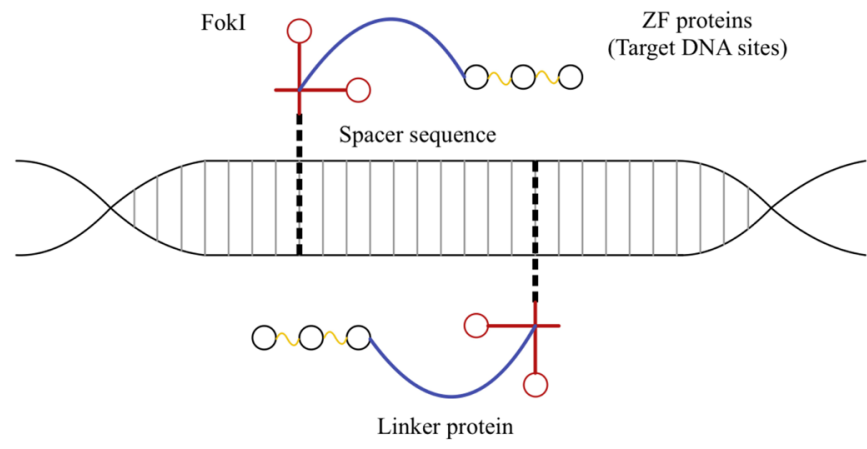

Figure 1: Structure of zinc-finger nuclease.

ZF: Zinc-finger

\section{Transcription Activator-Like Effector Nucleases}

Transcription activator-like effectors (TALEs) are a family of proteins regulating plant genome expression by binding to specific genes (21). These proteins are obtained from Xanthomonas bacteria, which contains pathogenic species for rice, pepper, tomato, wheat, and citrus (21-23). Xanthomonas bacteria increase the susceptibility of the plant cells to infection by secreting transcription activator-like effector (TALE) proteins to the cells' cytoplasm. TALE proteins are capable of imitating the eukaryotic transcription factors, thus activating the gene expression on the target DNA sites. Therefore, it is possible to increase, reduce and completely suppress gene expression by using TALEs. TALE proteins' genome modification activity was first demonstrated in 2007, and the code for TALE proteins to recognize the target DNA was deciphered a year later in 2008 (22). This advancement drew many researchers' attention to TALE proteins, deeming this technology worthy of being declared as the "Method of the Year" by Nature Methods in 2011 (24). Transcription activator-like effector nucleases' (TALENs) capability of editing endogenous genes in plants, nematodes, zebrafish, rats, human somatic cells, and pluripotent stem cells proves their versatility as a genome editing tool (25). Additionally, TALENs can be used for increasing crop yields, increasing the quality of agricultural products, and thus leading to a higher market share (21).

Similar to the ZFN system, the TALEN system consists of one DNA binding domain and one FokI catalytic domain (Figure 2) (26). Comparing with ZFN the TALEN system provides opportunities for replacement or inserting mutations at the specific genomic sequences of targeted genes with higher targeting flexibility, simplicity, and efficiency (27). Moreover, TALEs are highly sensitive to mismatches, blocking recognition after three to four mismatches and limiting the DNA binding capacity of TALENs (28). In order to cut the target area, a pair of TALENs that bind to both strands of DNA are required. The TALENs that are attached to either strand of DNA cannot function without being dimerized. These two TALENs must communicate with each other to perform nuclease activity since a single TALEN is not enough to induce a DSB. A 12-25 bp long spacer sequence between two adjacent TALENs is required. Once the dimerization process is completed, FokI nucleases induce DSBs at the targeted DNA sequence $(21,23)$. TALENs have lower off-target effects compared to other genome editing tools (29). Web-based tools are available that help researchers design pairs of TALENs to target specific gene sequences. Commonly used ones are TALE-NT and E-TALEN (21).

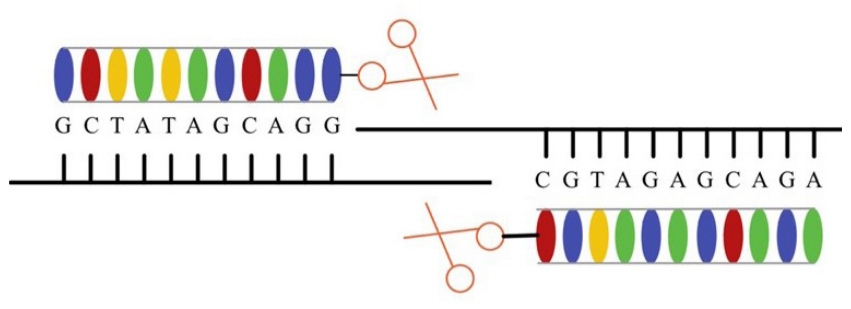

Figure 2: Structure of transcription activator-like effector nuclease.

The DNA binding domain of the TALEN system contains monomers, which are tandem repeats of 33 to 35 amino acids (26). Each monomer binds to one nucleotide that is present in the target DNA sequence. Being composed of only 20 amino acids, the last tandem repeat located in the 3 ' end is named a "half-repeat" (22). The 12th and 13th amino acids are highly variable and are called "repeat variable residues" (28). Repeat variable residues define the specificity of TALE proteins by recognizing specific nucleotides, while the repeat variable residue's DNA binding specificity is defined by the amino acids that match with these nucleotides.

The requirement of a T nucleotide before the 5 ' end of the target DNA sequence limits the site selection of TALEN regardless of its overall convenience and simplicity. However, there are two ways to overcome this limitation: site selection can either be made by altering the length of the spacer sequence or selecting the mutant variants of the TALEN N-terminal domain that are capable of binding to $\mathrm{A}, \mathrm{G}$, or $\mathrm{C}(23)$.

In a study conducted by Mussolino et al. (27), TALEN was reported to be able to successfully modify up to $45 \%$ of the transfected cells' CCR5 and interleukin-2 receptor subunit gamma loci. In the same study, a significantly lower rate of nuclease-associated cytotoxicity was reported for TALENs in comparison to ZFNs, indicating a higher specificity rate for TALENs. A study conducted by Sun et al. (30) reports that the TALEN system can potentially be used for curing sickle cell anemia by correcting the genetic mutation in the human beta-globin (HBB) gene that is responsible for this disease. Likewise, Bloom et al. (31) demonstrated TALEN's therapeutic potential against chronic HBV infection. TALEN has also been used in pigs for stopping the encoding of low-density lipoprotein receptors by inactivating the low-density lipoprotein receptor gene, which provided researchers with further understanding of familial hypercholesterolemia (32). This genome editing tool has immense therapeutic potential, tackling the adverse effects or the incidence of off-target mutations. 


\section{Clustered Regularly Interspaced Short Palindromic Repeats}

The clustered regularly interspaced short palindromic repeats (CRISPR) system is one of the defense mechanisms of prokaryotic organisms against viruses, making it possible for prokaryotic cells to memorize the invader's nucleic acid molecules and create an immune response. However, viruses have managed to counter this system by using random point mutations in their genetic material, more specifically, by creating point mutations in the protospacer adjacent motif (PAM) sequence or protospacer sequence. Bacterial populations with higher spacer diversity are less prone to being tricked by viruses that use point mutations (33).

The description of the CRISPR-Cas system was first disclosed in 1987 while analyzing the Escherichia coli genome sequence responsible for phosphate metabolism (34). However, the acronym CRISPR was not introduced until 2002 (35). Thereafter, similar sequences were reported in halophilic archaea and other bacteria (34). In 2005 , spacer sequences were found to have originated from bacteriophage genomes (36). Also, the PAM sequence was identified in the same year (35). Subsequently, it was suggested that the CRISPR system might be an adaptive immune defense against bacteriophages for prokaryotes. This suggestion was proved experimentally two years after the initial hypothesis (34). In 2008, it was shown that small and individual CRISPR RNAs (crRNAs) were produced transcribing CRISPR arrays that guide Cas enzymes. Researchers began understanding the mechanisms and fundamental functions of the CRISPR systems more clearly by 2010 (36). In 2011, trans-activating crRNA (tracrRNA) was identified, and it was discovered that tracrRNA and crRNA fuse to guide the Cas9 enzyme (37). In the following year, single guide RNA (sgRNA) was produced by merging crRNA and tracrRNA $(37,38)$. In 2013, genome editing in mammalian cells was achieved by using the CRISPR-Cas9 tool (37). Studies on cancer immunotherapy and treatment for blindness were initiated in the following years in the United States of America (38).

Although the history of clinical trials on genome editing date back to the 1960s, substantially more efficient and reliable techniques were introduced in 2012 when Jinek et al. (39) published their manuscript about the discovery of CRISPR-Cas9. Ultimately,
Emmanuelle Charpentier and Jennifer A. Doudna were awarded the Nobel Prize in Chemistry 2020 for pioneering the development of this revolutionary genome-editing tool that would allow scientists to "alter the code of life" (40).

As discoveries about the CRISPR systems accumulate, their classification has evolved to make it simpler (Figure 3) (34). Presently, CRISPR systems are divided into two classes, each containing three distinct types: class I uses a multiple-effector protein complex, whereas class II uses a single effector Cas protein to convey immunity against foreign nucleic acids by cleaving them. Each CRISPR system has different functions and characteristics, although the effects of the type IV CRISPR system on either of the nucleic acids are not yet known (Table 1) (41).

The signature protein for the type II CRISPR system, which was deemed worthy of the Nobel Prize in Chemistry 2020, is Cas9. Cas9 is a multi-domain protein responsible for binding the crRNA with the target sequence and cleaving the DNA (33). Two domains in Cas9 protein, named $\mathrm{HNH}$ and RuvC, break the opposite strands of DNA. While the HNH domain breaks the targeted strand of DNA, the RuvC domain breaks the complementary strand $(34,41)$. The type II CRISPR system essentially uses the Cas1, Cas2, and Cas9 enzymes along with tracrRNA and CRISPR array. While this set is sufficient for the type II-C system, type II-A and II-B systems require additional Csn 2 or Cas4 genes, respectively (35). The CRISPR Cas 9 system requires a sgRNA which can be designed by the association of a target sequence containing crRNA and tracrRNA. The sgRNA guides the Cas9 enzyme to find the PAM sequence and attach to the targeted area, causing a DSB $(35,42)$. Trans or transgenic DNA can be created by adding a donor piece between the two edges. The break in DNA will then be repaired by the cell. However, this technique renders the triggering of indels possible (38). The signature protein for the type III system is Cas10, and the PAM sequence is not required for this type (43).

The working principle of all CRISPR systems can be subclassified into three stages: adaptation (of spacer acquisition), expression (or crRNA biogenesis or maturation), and interference (Figure 4) $(33,44)$. Prokaryotic DNA contains a CRISPR locus, which consists of CRISPR and spacer parts (45). The length of the CRISPR sequence generally ranges from 28 nucleotides (nt) to $37 \mathrm{nt}$ although

Table 1: Function and domains of Cas and ancillary proteins.

\begin{tabular}{|c|c|c|}
\hline Cas Enzymes & CRISPR Type Association & Function \\
\hline Cas1 & I, II, some III, IV, possibly VI & DNA nuclease \\
\hline Cas2 & I, II, some III, V, some VI & RNA nuclease \\
\hline Cas3 & $\mathrm{I}$ & DNA nuclease, helicase \\
\hline Cas4 & Mostly I, II, V & DNA nuclease \\
\hline Cas5 & I, III, IV & Ribonuclease that turns pre-crRNA into crRNA \\
\hline Cas6 & Mostly I, some III & Ribonuclease that turns pre-crRNA into crRNA \\
\hline Cas7 & I, III, IV & Contains RNA recognition motif (RRM) and binds crRNA, generally present in multiple copies \\
\hline Cas8 & Mostly I & Large subunit of effector complex of type I \\
\hline Cas9 & II & DNA nuclease \\
\hline Cas10 & Some I, mostly III & Large subunit of effector complex of type III \\
\hline Cas12 (Cpf1) & $\mathrm{V}$ & DNA nuclease, processes crRNA \\
\hline Cas13 (C2c2) & VI & RNA nuclease, processes crRNA \\
\hline $\mathrm{Csm} / \mathrm{Cmr}$ & III & RNA nuclease, DNA nuclease (cleaves one strand) \\
\hline RNase III & II & Processes tracrRNA, assists crRNA maturation \\
\hline
\end{tabular}

CRISPR: Clustered regularly interspaced short palindromic repeats, Cas: CRISPR associated protein, DNA: Deoxyribonucleic acid, RNA: Ribonucleic acid, crRNA: CRISPR ribonucleic acid, tracrRNA: trans-activating CRISPR ribonucleic acid, RNase: Ribonuclease 
the minimum and maximum lengths are $23 \mathrm{nt}$ and $55 \mathrm{nt}$. The length of the spacer sequence generally ranges from $32 \mathrm{nt}$ to $38 \mathrm{nt}$; however, it can be as low as $21 \mathrm{nt}$ and as high as $72 \mathrm{nt}(46)$.

In the adaptation stage, the prokaryotic cell cuts off the protospacer from the invader's mobile genetic elements and adds this piece into its spacer DNA sequence between CRISPR systems, in order to deactivate the invader's mobile genetic elements more easily and faster in case of another encounter with the same invader. This process can be defined as "memorizing the invader's nucleic acid" $(33,45)$. The PAM sequence, which can be interpreted by its name, is located next to the protospacer, meaning that the prokaryotic cell can detect the PAM sequence along with the protospacer part. Protospacer detection with PAM sequence is only possible for types I and II (33). In this process, Cas1 (a DNA nuclease) and Cas2 (an RNA nuclease) enzymes are used, and these two proteins play a role in almost all CRISPR systems, with III-C, III-D, and IV being exceptions $(33,42)$. Both enzymes are responsible for cutting off the invader's nucleic acid in the adaptation stage $(33,46)$.

In the expression stage, the CRISPR locus in the microorganism's DNA is transcribed into precursor CRISPR RNAs (pre-crRNAs), which need to be maturated for activation. Cas proteins are needed for the maturation of the crRNAs containing the "memorized" sequences in the adaptation stage (44).

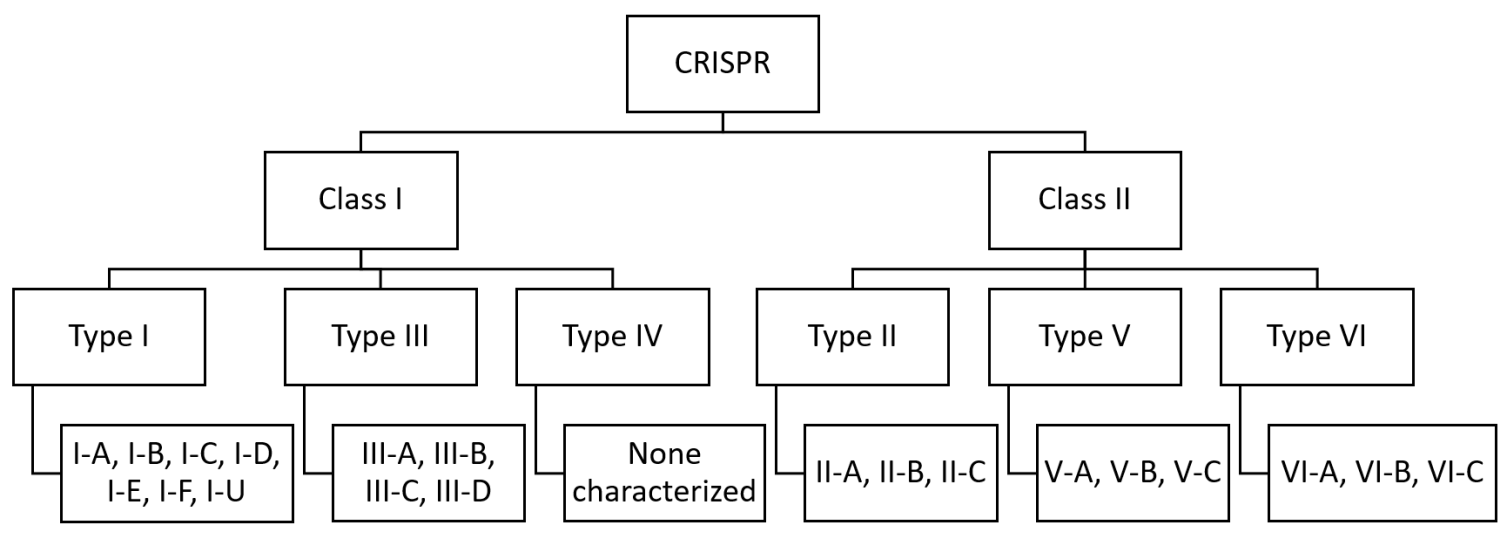

Figure 3: Classification of CRISPR systems.

CRISPR: Clustered regularly interspaced short palindromic repeats
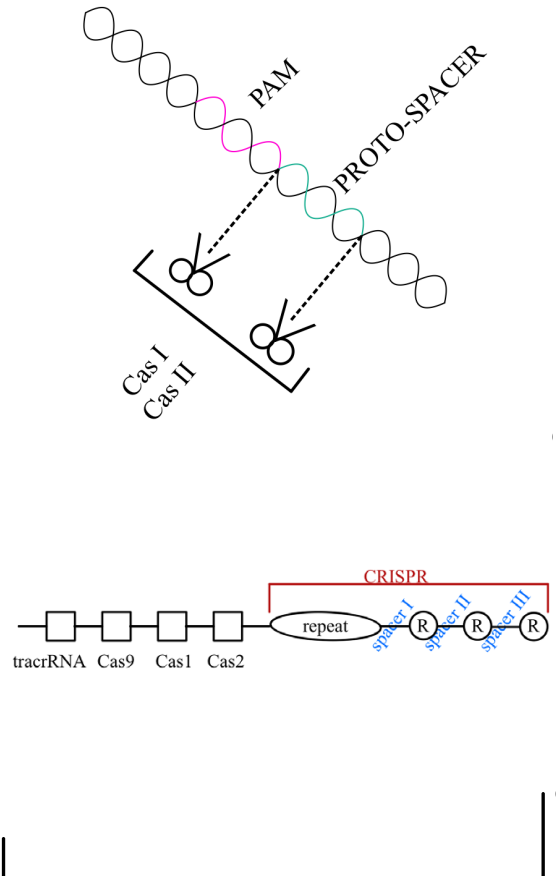

Adaptation
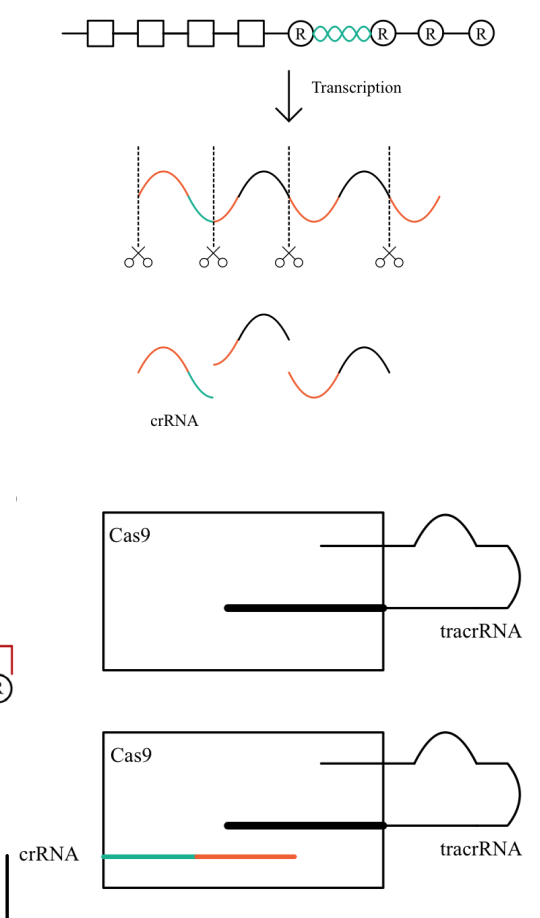

Expression
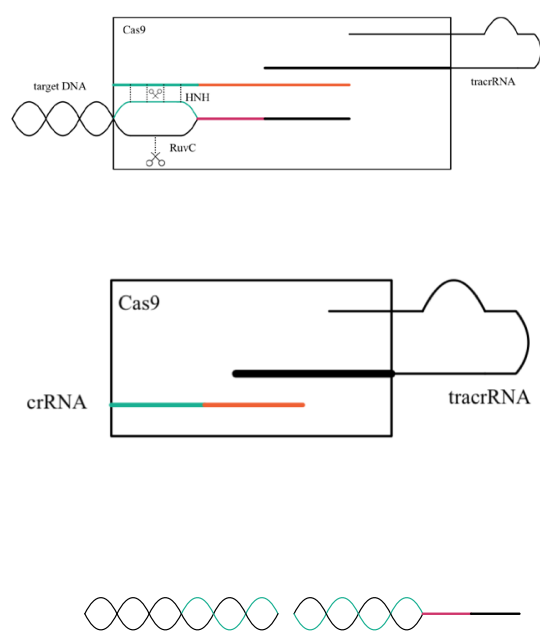

DSB

Figure 4: The working principle of clustered regularly interspaced short palindromic repeats systems.

CRISPR: Clustered regularly interspaced short palindromic repeats, crRNA: CRISPR RNA, tracrRNA: Trans-activating CRISPR RNA,

DSB: Double-strand berak, PAM: Protospacer adjacent motif, Cas: CRISPR associated protein 
In the interference stage, which is the last stage of immune reaction, crRNAs guide the Cas proteins to detect and interfere with the specific sequences. While class I CRISPR systems constitute a cascade complex (CRISPR-associated complex for antiviral defense), single effector proteins are sufficient for class II systems for interference. To avoid self-targeting, different CRISPR systems use different mechanisms. Self-targeting is avoided by recognizing the PAM sequence located upstream of the protospacer in types I and $\mathrm{V}$, while in type II it is avoided by the recognition of the PAM sequence located downstream of the protospacer. Mature crRNA in the type III system determines whether the immune response will be self-targeting or not. To proceed with the degradation process by the complex, the 5' tag of crRNA must not be paired with the target (33).

The principle of using the CRISPR tool for diagnostic purposes is "nucleic acids are indicators for diseases". This diagnostic method is based on identifying the disease-related nucleic acid sequences and cleaving them (47). The simplicity of the Type II system allows it to be used widely for diagnosis (42). Bacterial and viral sequences that are derived from infectious organisms or oncogenic mutations can be targeted. CRISPR systems not only identify pathogens but also repair the disease-causing genes on the chromosomes (47).

By using different characteristics of the CRISPR systems, several methods were developed for diagnostic purposes. These methods include diagnostic assay based on sgRNA, CRISPR interference, and serotyping/subtyping using CRISPR. The CRISPR interference method requires a catalytically-dead Cas9 (dCas9), which is a modified form of Cas9 that lacks endonucleolytic activity. A diagnostic assay based on sgRNA takes advantage of the specificity of the CRISPR technology. Since any sequence located next to the PAM sequence can be targeted, it is possible to detect almost any locus of interest by editing the sgRNA. The differentiation is of great importance, because specific strains may cause certain conditions, with one example being the Brazil strain of the Zika virus, which is associated with fetal microcephaly. CRISPR technology is being used for developing a paper diagnostic kit that can be used to distinguish between African and American Zika virus strains, despite the difference being a single base in the PAM sequence (42).

The effects of fluorescent probes can be increased by cleaving them from non-specific RNA with Cas13a, which is an RNA nuclease $(42,43)$. A molecular detection platform has been developed using this technique and named specific high sensitivity enzymatic reporter unlocking (SHERLOCK). This platform can be used to detect cell-free cancer mutations, single-nucleotide polymorphisms, viral strains, and bacterial pathogens (42). A similar platform using Cas12a named DNA endonuclease-targeted CRISPR trans reporter (DETECTR) can be used to diagnose various viral agents (47). A recently developed SARS-CoV-2 DETECTR reportedly has 95\% accuracy and requires around 40 minutes to detect COVID-19 (37).

Although CRISPR technology has immense potential for therapeutic application, some challenges still need to be overcome. Presently, the primary obstacle appears to be delivering the necessary enzymes and the donor DNA into the cell. Some alternatives to solve this problem include (i) using viral vectors to deliver the DNA sequence that encodes both guide RNA and enzymes, (ii) using lipid nanoparticles to deliver the mRNA that encodes guide RNA and Cas9, and (iii) preformed ribonucleoprotein included Cas9 and guide RNA.

The clustered regularly interspaced short palindromic repeats tool can be used for ex vivo based therapy by treating the selected cells of the organism and inserting them back with autologous transplantation. In order to cure immune-based or blood-based diseases, either hematopoietic stem or progenitor cells or immune cells need to be extracted from blood and bone marrow to be genetically edited (48). Vertex Pharmaceuticals and CRISPR Therapeutics offer a treatment named CTX001 for sickle-cell anemia and $\beta$-thalassemia using such a technique (37). However, editing stem cells or progenitor cells is not always necessary. For rheumatoid arthritis, editing regulatory $\mathrm{T}$ cells may be preferred instead of editing progenitor T cells (48).

Because of the minimal access to the targeted organs, in-vivo based therapy is less common. One of the targetable tissues for in-vivo based therapy is the eye. EDIT-101 is an alternative therapy for Leber congenital amaurosis caused by a mutation in the CEP290 gene with no known cure and results in childhood blindness. In this therapy, CRISPR/Cas9 is delivered into the patient's retina with the intronic IVS26 mutation. This mutation causes aberrant splicing on a specific protein. This splicing may be curable by using the CRISPR/Cas9 tool (37).

The first-ever phase 1 clinical trial using the CRISPR tool approved by the United States Food and Drug Administration has aimed at cancer immunotherapy by editing autologous $\mathrm{T}$ cells. This therapy was planned against several types of cancer without treatment options or with relapsed tumors, such as melanoma, multiple myeloma, myxoid/round cell liposarcoma, and synovial sarcoma. In this ex-vivo based therapy, $\alpha$ and $\beta$ chains are knocked out on endogenous $\mathrm{T}$ cell receptors, which generate an immune response, and programmed cell death protein 1 , which weakens the immune response. Later, edited genes are delivered back into the patients using lentivirus as a vector. The gene that encodes NY-ESO-1-specific T cell receptor has been demonstrated to be highly upregulated in the relapsed tumors (37).

Despite being prompt, economic, practical, and efficient, many challenges remain to be tackled until the CRISPR tool is available for widespread use (49). Toxicity is one of these disadvantages since DSBs created by CRISPR often trigger the apoptosis pathway of the cell. However, DSB risk may be reduced by using dCas9. Immunotoxicity is another phenomenon that often raises concerns. In a study conducted by Charlesworth et al. (38), more than $50 \%$ of the human subjects were found to possess pre-existing anti-Cas 9 antibodies. Similar to the other gene-editing technologies, the off-target effects of CRISPR are another major concern (11). The frequency of off-target effects in CRISPR is equal to or greater than 50\% (38). All of these drawbacks led researchers to conduct further studies in order to perfect this genome-editing tool. Various strategies have been developed to reduce RNA-guided endonucleases' off-target effects, namely Cas9 paired nickase, dCas9, CRISPR-based cytosine and adenine base editors, ribonucleoprotein delivery, truncated gRNAs, and prime editors $(50,51)$.

\section{After CRISPR: Prime Editing}

Effective and precise correction of most disease-causing gene variants using the ZFN, TALEN, and CRISPR technologies and other tools that produce DSBs is often hindered by excess byproducts and off-target mutations (52). In 2019, Anzalone et al. (52) from the Liu Lab introduced prime editing, a new genome editing tool that "promises to be a cut above CRISPR" and does not produce DSBs, thus having a lower off-target mutation rate (53). This novel genome-editing tool can correct targeted insertions, deletions, and all possible base-to-base conversions in comparison to CRISPR base editing, a genome-editing technology that targets cytosine and adenine for irreversible conversion without DSBs, which is capable of installing the four $C \rightarrow T, G \rightarrow A, A \rightarrow G$, and $T \rightarrow C$ mutations, rendering the mediation of all single nucleotide variations ( $\mathrm{SNV}$ ) possible (52, 54). It is anticipated that SNV correction will be a major focal point of precision medicine for years to come (54). 
Fundamentally, the prime editor structure consists of reverse transcriptase (RT) merged with Cas9n (an RNA-programmable nickase), along with a prime editing guide RNA (pegRNA) (Figure 5). Presently, there are four prime editing modifications: prime editor 1 (PE1), prime editor 2 (PE2), prime editor 3 (PE3), and prime editor $3 \mathrm{~b}$ (PE3b). The structure of PE1 comprises a nickase Streptococcus pyogenes Cas9 (SpCas9) merged with Moloney murine leukemia virus (M-MLV) RT and a pegRNA. PE2 utilizes a mutant variant of M-MLV RT and is a modification of PE1. When PE2 is modified by adding another nick on the opposite DNA strand, PE3 is formed. The fourth modification, PE3b, is formed when sequential double nicking is induced by the installation of the determined mutation on the targeted DNA sequence, nicking the non-edited DNA strand only after the conversion of the other strand to the edited sequence has been completed $(55,56)$.
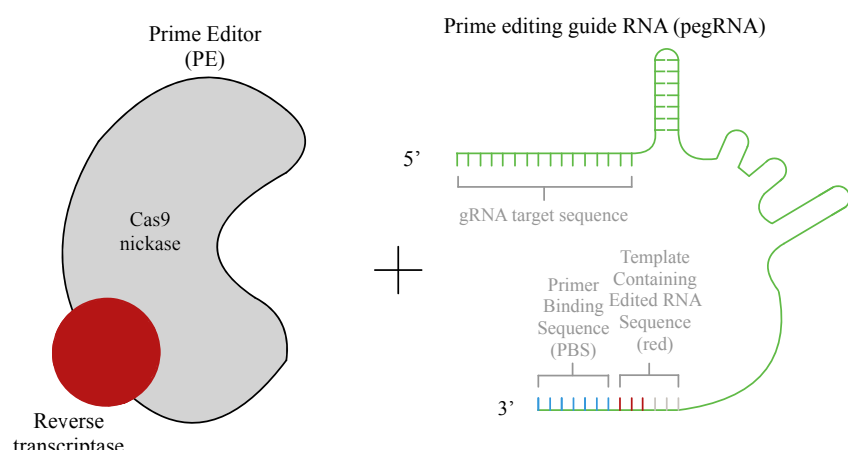

transcriptase

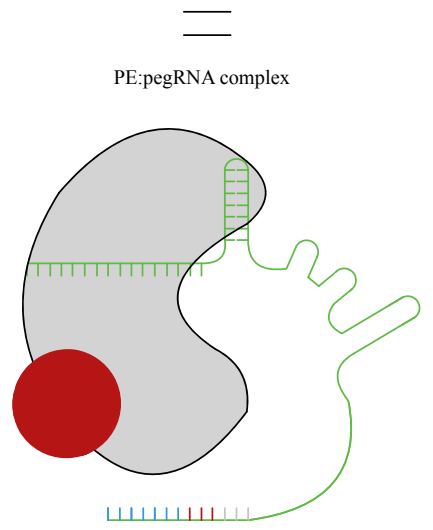

Figure 5: Structure of prime editors.

Prime editors do not produce DSBs, with PE3 creating two nicks and the other prime editors creating one nick. The absence of DSBs is associated with lower indel mutations, often at an undetectable level (55). Prime editing also offers several choices of different primer binding sites, pegRNA induced-nick locations, sgRNA induced second nick locations, and RT template lengths, along with the freedom to choose which DNA strand will be edited first. Allowing a wide range of gene modifications including insertions, deletions, and transversions, up to $89 \%$ of pathogenic human genetic variants may be potentially corrected with prime editing (54).

Despite showing immense therapeutic potential, more research is required for further understanding and more efficient use of prime editors. Although the working principles of CRISPR base editing are beyond the scope of this review, it is worth mentioning that base editing has been reported to outperform prime editing when using adenine base editors for correcting the pathogenic $\mathrm{G} \rightarrow \mathrm{A} \mathrm{mu-}$ tations in the ATP binding cassette subfamily B member-11 gene that causes bile salt export pump deficiency and the mutations of the serpin family A member-1 gene that causes alpha-1 antitrypsin deficiency (57). However, prime editors may be more favorable when multiple adenine and cytosine bases are present in the targeted DNA site and bystander edits are undesirable (52). Anzalone et al. (52) used PE3 to deploy the HBB (E6V) mutation causing sickle cell disease in the HEK293T cell line with 44\% efficiency and $4.8 \%$ indels. To revert this mutation to wild type $\mathrm{HBB}$, they treated the cell line with PE3 and a programmed pegRNA, with 26-52\% efficiency and $2.8 \%$ indels. Anzalone et al. (52) also tested prime editing to correct the mutation that causes Tay-Sachs disease, deploying a 4 bp insertion into hexosaminidase A by utilizing PE3 with $31 \%$ efficiency and $0.8 \%$ indels. In order to revert the cells to the wild type phenotype, PE3 was used, with the application resulting in $\geq 20 \%$ editing, compared to the application of PE $3 \mathrm{~b}$, which resulted in $33 \%$ efficiency and $0.32 \%$ indels. Prime editing has also been reported to be $7.1 \%$ more effective than CRISPR base editing on post-mitotic, terminally differentiated mice cells (52).

\section{Ethical Questions Regarding Genome-Editing}

Ethical and regulatory issues regarding the use of genome editing tools are being discussed globally and many guidelines, rules, and principles to advance the usage of these technologies are being accepted. Current standards and guidelines are not sufficient for the safe use of these applications, especially for editing the human genome. Since these novel technologies have not been thoroughly tested on editing the human genome, many unpredictable complications may occur during and after application (8). Current issues present mental, cultural, and philosophical conflicts for researchers, clinical specialists, strategy producers, patients, and the general public (54).

Presently, the CRISPR-Cas technique is a practical option for genome alteration in a wide variety of organisms, including humans, where this method has been tried for treating or preventing severe genetic defects. The CRISPR-Cas method can be exceptionally favorable in basic and pre-clinical settings to distinctly explain and further improve the application itself to be relevant in clinical search. However, a significant matter of concern about the CRISPR-Cas method is its potential utilization in human embryo germline editing, as the debate on precisely when an embryo accomplishes "personhood" is yet to be concluded (8).

During the 2015 Napa Valley meeting, a group of developers, scientists, and ethicists from the United States National Academy of Sciences, Engineering and Medicine (NASEM) discussed the legal, ethical, and biomedical aspects of using gene editing methods without sacrificing the benefits of discoveries on the CRISPR-Cas9 mechanism (8). In the follow-up meeting held in February 2017, NASEM declared supporting somatic genome editing, excluding any enhancement. The committee has also decided that the alteration of a germ-line in order to create new individuals who might potentially transfer the altered genome to their off-springs could be allowed under particular conditions, being: (i) treating severe genetic defects in the absence of an alternative approach or treatment, under meticulous supervision, (ii) somatic and germ cells cannot be edited with the intent of enhancement, (iii) genome editing can be done for basic research, and (iv) somatic genome editing can be used for the treatment of severe diseases (58).

In November 2018, Chinese researcher He Jiankui declared that he had used CRISPR to edit human embryos by causing a 32 bp deletion on the CCR5 gene, aiming to make the embryos resistant to AIDS by rendering the associated gene dysfunctional. Two of these edited embryos were successfully implanted, resulting in pregnancy and birth by cesarean section. Although Jiankui expected to be "hailed as a hero", in reality, he was condemned by the scientific 
community and his work was defined as "premature and unethical". The additional effects of the absence of a functional CCR5 gene are currently unknown, and whether Jiankui managed to successfully induce the $32 \mathrm{bp}$ deletion on either of the twins is a matter of controversy (59).

Editing human embryos has long been among the greatest ethical concerns of the CRISPR genome-editing system, and the "Jiankui Affair" may have further fueled the ongoing debate. One reason for this concern is the possibility that any damage done to the embryonic genome that causes mutations and off-target effects can be transferred to future generations as well (60). While many scientific community members support fundamental search on CRISPR in somatic cells, a significant number of researchers consider the CRISPR tool to be not extensively developed to insert hereditary substitutions in the human genome (61).

Different countries have prepared restrictive rules and guidelines for human germline gene editing for reproductive purposes. These guidelines diverge widely around the world, placing germline genome editing on a spectrum of limitations ranging from directly "banning" to "outlawing in any circumstances" around the world (Table 2) (8).

Table 2: Legal perception of human germ-line editing in various countries (8).

\begin{tabular}{|c|c|c|c|c|}
\hline Country & Restrictive & $\begin{array}{c}\text { Legal } \\
\text { prohibition }\end{array}$ & $\begin{array}{c}\text { Prohibition } \\
\text { by guidelines }\end{array}$ & Ambiguous \\
\hline $\begin{array}{l}\text { United States of } \\
\text { America }\end{array}$ & $\mathrm{X}$ & & & \\
\hline United Kingdom & & $\mathrm{X}$ & & \\
\hline Japan & & & $\mathrm{X}$ & \\
\hline China & & & $\mathrm{X}$ & \\
\hline Ireland & & & $\mathrm{X}$ & \\
\hline India & & & $\mathrm{X}$ & \\
\hline Russia & & & & $\mathrm{X}$ \\
\hline Argentina & & & & $\mathrm{X}$ \\
\hline South Africa & & & & $\mathrm{X}$ \\
\hline Chile & & & & $\mathrm{X}$ \\
\hline Slovakia & & & & $\mathrm{X}$ \\
\hline Colombia & & & & $\mathrm{X}$ \\
\hline Greece & & & & $\mathrm{X}$ \\
\hline Iceland & & & & $\mathrm{X}$ \\
\hline Peru & & & & $\mathrm{X}$ \\
\hline
\end{tabular}

In many countries, biotechnological policies are superannuated and the genome editing policies regarding the use of such tools for clinical, reproductive, and agricultural purposes are poorly discussed. The rapid discoveries on novel genome editing tools, especially the recent advancements on the CRISPR method, render the existing moral guidelines and regulatory policies requiring further discussion and reconsideration to reach a global consensus on the ethical aspects of human genome editing. Participants' privacy, well-being, safety, and dignity should be the number one priority of these discussions (8).

\section{CONCLUSION}

Precise and effective genome modification is of immense value for genetic engineering. The rapid development of such technologies makes it possible for researchers to use an arsenal of ever-expanding techniques for genome editing (58). TALENs and ZFNs were the pioneering genome editing tools; however, they do not offer the highest specificity due to their substantial off-target effects. With the discovery of CRISPR-Cas9, genome editing has become more incentivized as a result of providing higher efficiency and applicability. This recent technology appears to be accepted as the "new level" of genome editing (62).

Compared to other preceding programmable gene-editing technologies such as TALENs and ZFNs, the CRISPR tool comes into prominence due to its simplicity and lower cost, making it more readily available for research communities (58). Only a short guide RNA sequence is required to be changed for redirecting the site-specific cleavage, which may also be turned into an enzyme that nicks to simplify homology-directed repair with lower mutagenic activity $(58,63)$. The high effectiveness and accuracy of the Cas9 protein derived from the type II CRISPR system allows several applications in numerous fields of science (58).

In contrast to the ZFN and TALEN systems in which the DNA recognition sites are dependent on the artificial proteins that require an interaction between the protein and DNA, the DNA recognition function of the CRISPR/Cas system depends on RNA-DNA coactions, presenting some advantages over ZFNs and TALENs. This feature provides a simple design for altering any genomic target, more predictable off-target regions, and the prospect of modifying multiple genomic regions concurrently (11).

The high probability of undesirable off-target effects during gene editing appears to be the most prominent disadvantage of the ZFN technology. Being structurally similar to ZFN, the TALEN system also suffers from high rates of undesirable mutations in the target site. Being an RNA-guided nuclease, Cas9 has a higher sequence specificity owing to Watson-Crick base pairing between the target DNA sequence and its gRNA, in addition to the direct connection between Cas9 and PAM. Although ZFNs' and TALENs' effectiveness for certain purposes has been acknowledged, these tools require new proteins to be synthesized for every new target DNA site. Contrarily, the Cas9 protein maintains the same structure, regardless of which DNA sequence is targeted. Only the short sequence of gRNA needs to be altered to redirect the site-specific cleavage (58). However, all three of these genome editing tools induce DSBs, the ultimate risk factor for off-target mutations. Prime editing developed by Anzalone et al. (52) generates mere nicks on the target sequence rather than DSBs, which may eventually rid the researchers of this "necessary evil" of genome editing.

Even though these genome editing technologies promise countless benefits, hopes of treatment for serious diseases with their immense therapeutic potential, and the power to utterly "alter the code of life", significant ethical and biosafety issues should not be ignored (58). The Jiankui affair may have greatly altered how researchers perceive genome editing by adding more questions to be answered to an already controversial topic. This incidence clearly indicates that many regulatory rules and perhaps decades of further studies are required before the human genome can be safely edited. However, the answer to the ultimate question of whether we should edit the human genome is yet to be given. 
Ethics Committee Approval: N/A

Informed Consent: N/A

Conflict of Interest: The authors declared no conflict of interest.

Author Contributions: Concept: EŞ, DKȘ, BRA, AD, SS, GFA, SD. Design: EŞ, DKŞ, BRA, AD, SS, GFA, SD. Supervision: EŞ, DKŞ, BRA, AD, SS, GFA, SD. Data collection and/or processing: ES, DKS, BRA, AD, SS, GFA, SD. Analysis and/or Interpretation: EŞ, DKŞ, BRA, AD, SS, GFA, SD. Literature Search: EŞ, DKŞ, BRA, AD, SS, GFA, SD. Writing Manuscript: EŞ, DKŞ, BRA, AD, SS, GFA, SD. Critical Review: EŞ, DKŞ, BRA, AD, SS, GFA, SD.

Financial Disclosure: The authors declared that this study received no financial support.

Editor-in-Chief's Note: One of the authors of this article, Eylül Şenödeyici is member of the editorial board of Turkish Medical Student Journal. However, she did not take place at any stage of the editorial decision of the manuscript. The editors who evaluated this manuscript are from other institutions.

\section{REFERENCES}

1. Szybalska EH, Szybalski W. Genetics of human cell lines, iv. DNA-mediated heritable transformation of a biochemical trait. Proc Natl Acad Sci USA 1962;48(12):2026-34.

2. Redwan ERM, Matar SM, El-Aziz GA et al. Synthesis of the human insulin gene: protein expression, scaling up and bioactivity. Prep Biochem Biotechnol 2008;38(1):24-39.

3. Espejo-Mojica ÁJ, Alméciga-Díaz CJ, Rodríguez A et al. Human recombinant lysosomal enzymes produced in microorganisms. Mol Genet Metab 2015;116(12):13-23.

4. Lander ES, Linton LM, Birren B et al. Initial sequencing and analysis of the human genome. Nature 2001;409(6822):860-921.

5. International Human Genome Sequencing Consortium. Finishing the euchromatic sequence of the human genome. Nature 2004;431(7011):931-45.

6. Hood L, Rowen L. The human genome project: big science transforms biology and medicine. Genome Med 2013;5(9):79.

7. Cring MR, Sheffield VC. Gene therapy and gene correction: targets, progress, and challenges for treating human diseases. Gene Ther 2020 Oct 9. doi: 10.1038/ s41434-020-00197-8. [Epub ahead of print]

8. Zhang D, Hussain A, Manghwar $\mathrm{H}$ et al. Genome editing with the CRISPRCas system: an art, ethics and global regulatory perspective. Plant Biotechnol J 2020;18(8):1651-69.

9. Eid A, Mahfouz MM. Genome editing: the road of CRISPR/Cas9 from bench to clinic. Exp Mol Med 2016;48(10):e265.

10. Coller BS. Ethics of human genome editing. Annu Rev Med 2019;70(1):289-305.

11. Khalil AM. The genome editing revolution: review. J Genet Eng Biotechnol 2020;18(1):68.

12. Carroll D. Genome engineering with zinc-finger nucleases. Genetics 2011;188(4):773-82.

13. Wright DA, Thibodeau-Beganny S, Sander JD et al. Standardized reagents and protocols for engineering zinc finger nucleases by modular assembly. Nat Protoc 2006;1(3):1637-52.

14. Urnov FD, Miller JC, Lee YL et al. Highly efficient endogenous human gene correction using designed zinc-finger nucleases. Nature 2005;435(7042):646-51.

15. Sander JD, Dahlborg EJ, Goodwin MJ et al. Selection-free zinc-finger-nuclease en gineering by context-dependent assembly (CoDA). Nat Methods 2011;8(1):67-9.

16. Tebas P, Jadlowsky JK, Shaw PA et al. CCR5-edited CD4+ T cells augment HIV-specific immunity to enable post-rebound control of HIV replication. J Clin Invest 2021;131(7):e144486.

17. Stone D, Niyonzima N, Jerome KR. Genome editing and the next generation of antiviral therapy. Hum Genet 2016;135(9):1071-82.

18. Weber ND, Stone D, Sedlak RH et al. AAV-mediated delivery of zinc finger nucleases targeting hepatitis B virus inhibits active replication. PLoS One 2014;9(5):e97579.

19. Nain V, Sahi S, Verma A. CPP-ZFN: a potential DNA-targeting anti-malarial drug. Malar J 2010;9:258.

20. Kim HJ, Lee HJ, Kim $\mathrm{H}$ et al. Targeted genome editing in human cells with zinc finger nucleases constructed via modular assembly. Genome Res 2009;19(7):1279-88.

21. Akbudak MA, Kontbay K. Yeni nesil genom düzenleme teknikleri: ZFN, TALEN, CRISPR'lar ve bitkilerde kullanımı. Tarla Bitkileri Merkez Araştırma Enstitüsü Dergisi 2017;26(1):111-26.

22. Nemudryi AA, Valetdinova KR, Medvedev SP et al. TALEN and CRISPR/Cas genome editing systems: tools of discovery. Acta Naturae 2014;6(3):19-40.

23. Timilsina S, Potnis N, Newberry EA et al. Xanthomonas diversity, virulence and plant-pathogen interactions. Nat Rev Microbiol 2020;18(8):415-27.

24. Method of the year 2011. Nat Methods 2012;9(1):1.

25. Reyon D, Tsai SQ, Khayter C et al. FLASH assembly of TALENs for high-throughput genome editing. Nat Biotechnol 2012;30(5):460-5.

26. Budhagatapalli N, Rutten T, Gurushidze M et al. Targeted modification of gene function exploiting homology-directed repair of TALEN-mediated double-strand breaks in barley. G3 2015;5(9):1857-63.
27. Mussolino C, Morbitzer R, Lütge F et al. A novel TALE nuclease scaffold enables high genome editing activity in combination with low toxicity. Nucleic Acids Res 2011;39(21):9283-93.

28. Scholze H, Boch J. TAL effectors are remote controls for gene activation. Curr Opin Microbiol 2011;14(1):47-53.

29. Kadam US, Shelake RM, Chavhan RL et al. Concerns regarding 'off-target' activity of genome editing endonucleases. Plant Physiol Biochem 2018;131:22-30.

30. Sun N, Liang J, Abil Z et al. Optimized TAL effector nucleases (TALENs) for use in treatment of sickle cell disease. Mol Biosyst 2012;8(4):1255-63.

31. Bloom K, Ely A, Mussolino C et al. Inactivation of hepatitis B virus replication in cultured cells and in vivo with engineered transcription activator-like effector nucleases. Mol Ther 2013;21(10):1889-97.

32. Carlson DF, Tan W, Lillico SG et al. Efficient TALEN-mediated gene knockout in livestock. Proc Natl Acad Sci USA 2012;109:17382-7.

33. Hille F, Charpentier E. CRISPR-Cas: biology, mechanisms and relevance. Philos Trans R Soc Lond B: Biol Sci 2016;371(1707):20150496.

34. Ishino Y, Krupovic M, Forterre P. History of CRISPR-Cas from encounter with a mysterious repeated sequence to genome editing technology. J Bacteriol 2018;200(7):e00580-17.

35. Hsu PD, Lander ES, Zhang F. Development and applications of CRISPR-Cas9 for genome engineering. Cell 2014;157(6):1262-78.

36. Javed MR, Sadaf M, Ahmed T et al. CRISPR-Cas system: history and prospects as a genome editing tool in microorganisms. Curr Microbiol 2018;75(12):1675-83.

37. Uddin F, Rudin CM, Sen T. CRISPR gene therapy: applications, limitations, and implications for the future. Front Oncol 2020;10(7):1387.

38. Costa JR, Bejcek BE, McGee JE et al. Genome editing using engineered nucleases and their use in genomic screening. Assay Guidance Manual (serial online) 2017 Nov 20 (cited 2021 Mar 8) : (23 screens). Available from: URL: https://www.ncbi.nlm.nih.gov/books/NBK464635/.

39. Jinek M, Chylinski K, Fonfara I et al. A programmable dual-RNA-guided DNA endonuclease in adaptive bacterial immunity. Science 2012;337(6096):816-21.

40. Strzyz P. CRISPR-Cas9 wins Nobel. Nat Rev Mol Cell Biol 2020;21(12):714

41. Liu Z, Dong H, Cui Y et al. Application of different types of CRISPR/Cas-based systems in bacteria. Microb Cell Fact 2020;19(1):172.

42. Uppada V, Gokara M, Rasineni GK. Diagnosis and therapy with CRISPR advanced CRISPR based tools for point of care diagnostics and early therapies. Gene 2018;656:22-9.

43. Clark DP, Pazdernik NJ, McGehee MR. Genome Defense. In: Clark DP, Pazdernik NJ, McGehee MR, editors. Molecular Biology (Third Edition). London: Academic Press; 2019.p.622-53.

44. Makarova KS, Wolf YI, Alkhnbashi OS et al. An updated evolutionary classification of CRISPR-Cas systems. Nat Rev Microbiol 2015;13(11):722-36.

45. Makarova KS, Haft DH, Barrangou R et al. Evolution and classification of the CRISPR-Cas systems. Nat Rev Microbiol 2011;9(6):467-77.

46. Barrangou R, Marraffini LA. CRISPR-Cas systems: prokaryotes upgrade to adaptive immunity. Mol Cell 2014;54(2):234-44.

47. Jolany Vangah S, Katalani C, Boone HA et al. CRISPR-Based diagnosis of infectious and noninfectious diseases. Biol Proced Online 2020;22(1):22.

48. Foss DV, Hochstrasser ML, Wilson RC. Clinical applications of CRISPR-based genome editing and diagnostics. Transfusion 2019;59(4):1389-99.

49. Gupta D, Bhattacharjee O, Mandal D et al. CRISPR-Cas9 system: a new-fangled dawn in gene editing. Life Sci 2019;232:116636.

50. Zhang XH, Tee LY, Wang XG et al. Off-target effects in CRISPR/Cas9-mediated genome engineering. Mol Ther Nucleic Acids 2015;4(11):e264.

51. Naeem M, Majeed S, Hoque MZ et al. Latest developed strategies to minimize the off-target effects in CRISPR-Cas-mediated genome editing. Cells 2020;9(7):1608.

52. Anzalone AV, Randolph PB, Davis JR et al. Search-and-replace genome editing without double-strand breaks or donor DNA. Nature 2019;576(7785):149-57.

53. Cohen J. Prime editing promises to be a cut above CRISPR. Science 2019;366(6464):406.

54. Porto EM, Komor AC, Slaymaker IM et al. Base editing: advances and therapeutic opportunities. Nat Rev Drug Discov 2020;19(12):839-59.

55. Anzalone AV, Koblan LW, Liu DR. Genome editing with CRISPR-Cas nucleases, base editors, transposases and prime editors. Nat Biotechnol 2020;38(7):824-44.

56. Schene IF, Joore IP, Oka R et al. Prime editing for functional repair in patient-derived disease models. Nat Commun 2020;11(1):5352.

57. Smirnikhina SA. Prime editing: making the move to prime time. CRISPR J 2020;3(5):319-21.

58. Janik E, Niemcewicz M, Ceremuga $\mathrm{M}$ et al. Various aspects of a gene editing system-CRISPR-Cas9. Int J Mol Sci 2020 16;21(24):9604.

59. Greely HT. CRISPR'd babies: human germline genome editing in the 'He Jiankui affair'. J Law Biosci 2019;6(1):111-83.

60. Bosley KS, Botchan M, Bredenoord AL et al. CRISPR germline engineering - the community speaks. Nat Biotechnol 2015;33(5):478-86.

61. Lanphier E, Urnov F, Haecker SE et al. Don't edit the human germ line. Nature 2015;519(7544):410-1.

62. Khan SH. Genome-editing technologies: concept, pros, and cons of various genome-editing techniques and bioethical concerns for clinical application. Mol Ther Nucleic Acids 2019;16:326-34.

63. Cong L, Ran FA, Cox D et al. Multiplex genome engineering using CRISPR/Cas systems. Science 2013;339(6121):819-23. 